\title{
EI derecho a la educación y los conflictos armados: Cuando la violencia opaca la esperanza y el desarrollo(*)
}

\author{
The Right to Education and Armed Conflict: When Violence Obscures Hope \\ and Development
}

\author{
Dante Martin Paiva Goyburu(*) \\ Universidad Nacional Mayor de San Marcos (Lima, Perú)
}

Resumen: El presente trabajo analiza las lesiones que se configuran contra el derecho fundamental a la educación en el marco de los conflictos armados. Para ello se hace un repaso de los alcances y contenido del derecho fundamental, así como de la situación de los conflictos armados en el presente siglo, donde algunos enfrentamientos parecen perennizados. Como tal, se ha determinado que los conflictos armados y el perjuicio que cometen contra el proceso educativo terminan afectando a toda la comunidad educativa, siendo los estudiantes los que llevan la peor parte. Asimismo, la situación involucra tanto a agentes pasivos como también, en el caso de los menores, cuando estos últimos intervienen de forma activa como "niños soldado". Finalmente, se resalta la urgencia de adoptar medidas más efectivas en torno a la problemática, teniendo en cuenta que en el Perú, debido a los conflictos en el VRAEM, la situación se encuentra presente, afectando a un sector vulnerable que ve su integridad constantemente en riesgo, pero también se alejan de cualquier oportunidad de progreso al verse truncado su acceso a la educación. Las políticas a adoptarse demandan todo el apoyo y esfuerzo de la comunidad nacional e internacional.

Palabras clave: Derechos fundamentales - Educación - Conflictos armados - Docentes - Estudiantes

\begin{abstract}
This work analyzes the injuries that are configured against the fundamental right to education in the context of armed conflicts. For this, a review is made of the scope and content of the fundamental right, as well as the situation of armed conflicts in this century, where some confrontations seem perennial. As such, it has been determined that armed conflicts and the damage they commit against the educational process end up affecting the entire educational community, with students bearing the brunt. Likewise, the situation involves both passive agents and also, in the case of minors, when the latter actively intervene as "child soldiers". Finally, the urgency of adopting more effective measures around the problem is highlighted, taking into account that in Peru, due to the conflicts in the VRAEM, the situation is present, affecting a vulnerable sector that constantly sees its integrity in risk, but they also shy away from any opportunity for advancement as their access to education is truncated. The policies to be adopted require all the support and efforts of the national and international community.
\end{abstract}

Keywords: Fundamental rights - Education - Armed conflicts - Teachers - Students

(*) Nota del Equipo Editorial: Este artículo fue recibido el 26 de octubre de 2021 y su publicación fue aprobada el 30 de noviembre de 2021.

$\left({ }^{* *}\right)$ Doctor en Derecho y Ciencia Política por la Universidad Nacional Mayor de San Marcos. Secretario de Redacción de la Revista Peruana de Derecho Público. Miembro de la Asociación Peruana de Derecho Constitucional y del Instituto Iberoamericano de Derecho Constitucional - Sección Peruana. Investigador CONCYTEC (Grupo Carlos Monge). ORCID: https://orcid.org/0000-0001-9140-6580. Correo electrónico: dante.paiva@unmsm.edu.pe. 


\section{Introducción}

Frente a los conflictos armados que, con variaciones y distintos alcances, son una constante en la humanidad, los derechos fundamentales siempre se han visto en riesgo.

A partir de ello, advertimos que el derecho a la educación se ve particularmente afectado por el desarrollo de estos enfrentamientos, perjudicando en gran medida a los agentes del servicio educativo, y generando un daño muy profundo, no solo durante el desarrollo de los enfrentamientos, sino en especial de las secuelas y la posibilidad de recuperar el tiempo perdido.

De esta forma, debe considerarse que un genuino respeto al derecho humano a la educación exige que bajo ninguna circunstancia el acceso a la enseñanza y el aprendizaje se vea limitado, en especial para los niños, cuya labor primordial es adquirir conocimientos y formación.

Como tal, con el propósito de apoyar en la formulación de medidas de carácter político y humanitario, corresponde realizar un análisis sobre las consecuencias de los conflictos armados en el ejercicio del derecho a la educación, procurando brindar soluciones y elaborar propuestas concretas que puedan ser empleadas, comprometiendo a las autoridades locales así como a la comunidad internacional y puedan realizar acciones con eficacia para revertir el desmedro que padecen a nivel mundial los escolares producto de los conflictos.

En síntesis, mediante el presente estudio procuraremos responder a la siguiente pregunta ¿Qué lesiones configuran los conflictos armados contra el derecho fundamental a la educación y qué medidas son necesarias para afrontar las mismas?

\section{El derecho a la educación}

Las tendencias globales con el gran impulso surgido a partir de la sociedad de la información deparaban grandes expectativas, toda vez que se contaba con mejores condiciones, inéditas, para poder difundir el saber, sumándose ahora los canales virtuales y alcanzar así lugares remotos, superando las usuales barreras geográficas. Así podía accederse a contenido actualizado sobre diversos tópicos y categorías.

Todo este ámbito redimensiona el derecho a la educación. Al respecto, debemos recordar que el concepto actual del derecho a la educación implica que todo ser humano debe contar para sí, obligatoria y gratuitamente, con educación primaria y también accesibilidad para la secundaria y la educación universitaria. Esto es producto de movimientos civiles y políticos, que se encuentran refrendados legislativamente, a través de normas nacionales y acuerdos multilaterales. Esto va de la mano con la necesidad de erradicar el analfabetismo a nivel mundial y que por medio del conocimiento puedan revertirse la pobreza y las desigualdades sociales. El derecho a la educación se concreta, en tres aspectos fundamentales: un marco regulatorio, objetivos educacionales y en la prestación de un servicio, sobre lo cual se precisa:

El derecho a la educación se constituye hoy, en países como el nuestro, en una exigencia concomitante del principio de legitimidad democrática del Estado y en una condición imprescindible para la efectividad del derecho al libre desarrollo de la personalidad (García Toma, 2008, p. 380).

Sin embargo, si bien contamos en el Perú y América Latina con un sistema jurídico basado en instituciones romanísticas, germánicas, el respeto y exigencia de este derecho no se da uniformemente en todos los países con el mismo sistema. En el caso peruano, hay condiciones que vician en gran medida que el derecho a la educación su cumpla a cabalidad conforme las condiciones que se esperan, en ese sentido se afirma:

Toda la opinión al respecto sugiere que a pesar de que el país cuenta con una sólida plataforma legal sobre el derecho a la educación y que por lo tanto es un deber de los ciudadanos exigirlo al Estado, no queda del todo claro qué es lo que se debe reclamar. Por otro lado, a pesar de que en el ámbito jurídico se ha reconocido el derecho a la educación, ese acto no ha tenido el impacto esperado. Esto último ha sido atribuido tanto a factores externos al sistema educativo, de corte más estructural, como a factores internos del sistema y a la manera como se ha enunciado el derecho a la educación en las políticas educativas peruanas (Cuenca, 2012, p. 26).

Hay que rescatar la verdadera trascendencia e importancia del derecho a la educación, el cual es un derecho humano, de carácter universal, debiendo adoptar las acciones para su respeto en todo el mundo, así:

Garantizar la vigencia del Derecho Humano a la Educación significa que todos los involucrados (niños, jóvenes, adultos) tengan posibilidad de acceder a lo que la UNESCO, en la Reunión de Ministros de Educación de Todo el Mundo, realizada en Tailandia, definió como las Necesidades Básicas de Aprendizaje. 
Esta definición hace referencia a un conjunto de herramientas esenciales para el aprendizaje y de los contenidos básicos de aprendizaje (conocimientos prácticos y teóricos, valores y actitudes) necesarios para que los seres humanos puedan sobrevivir, desarrollar plenamente sus capacidades, vivir y trabajar con dignidad, participar plenamente en el desarrollo, mejorar la calidad de vida, tomar decisiones fundamentadas y continuar aprendiendo (Declaración Mundial sobre una Educación para todos - UNESCO, 1990) (Nicoletti, 2009, pp. 79-80).

Precisamente, la garantía sobre este derecho se encuentra prevista tanto en la Declaración Universal de los Derechos Humanos, el Pacto de los Derechos Económicos, Sociales y Culturales y las constituciones de los diversos países del mundo (entre ellos el Perú). En este sentido, dentro de las implicancias del derecho fundamental a la educación se considera:

a) El reconocimiento y garantía por parte del Estado de la libertad de enseñanza.

b) La educación no debe ser exclusiva del Estado, ni de la promoción del desarrollo científico y tecnológico del país.

c) La obligatoriedad y gratuidad (en las instituciones del Estado) de la educación inicial, primaria y secundaria; y,

d) La obligatoriedad, por parte del Estado, de coordinar la política educativa (Chanamé Orbe, 2011, pp. 334 - 335).

A nivel jurisprudencial, el Tribunal Constitucional peruano ha realizado aportes sobre el contenido constitucionalmente protegido dentro del derecho a la educación, señalando que éste corresponde a:

(...) el acceso a una educación adecuada (artículo 16), la libertad de enseñanza (artículo 13), la libre elección del centro docente (artículo 13), el respeto a la libertad de conciencia de los estudiantes (artículo 14), el respeto a la identidad de los educandos, así como a un buen trato psicológico y físico (artículo 15), la libertad de cátedra (artículo 18), y la libertad de creación de centros docentes y universidades (artículos 17 y 18) (Expediente 0091-2005-PA/TC, fundamento 6).

En otra sentencia posterior, el Tribunal Constitucional ha prescrito las manifestaciones que se producen de este derecho, a saber:

a) El acceder a una educación, el cual tiene dos aristas fundamentales: a.1) Cobertura educativa y a.2) El acceso a la educación en sentido estricto;

b) La permanencia y el respeto a la dignidad del escolar [art. 15 segundo párrafo]; $y$,

c) La calidad de la educación [art. 15 primer párrafo y 16 segundo párrafo], asimismo precisará que estas tres manifestaciones conforman la estructura básica del derecho a la educación (Expediente 4646-2007-AA/TC, pp. 8-11)

Dentro de las fuentes del derecho a la educación encontramos en nuestra legislación interna a la Constitución y a la Ley 28044 - Ley General de Educación, así como a reglamentos y directivas aprobadas por decretos y resoluciones del sector correspondiente, respectivamente.
En este sentido, cabe destacar que los fines de la educación han sido precisados en el artículo 9 de la Ley 28044 en los términos siguientes:

a) Formar personas capaces de lograr su realización ética, intelectual, artística, cultural, afectiva, física, espiritual y religiosa, promoviendo la formación y consolidación de su identidad y autoestima y su integración adecuada y crítica a la sociedad para el ejercicio de su ciudadanía en armonía con su entorno, así como el desarrollo de sus capacidades y habilidades para vincular su vida con el mundo del trabajo y para afrontar los incesantes cambios en la sociedad y el conocimiento.

b) Contribuir a formar una sociedad democrática, solidaria, justa, inclusiva, próspera, tolerante y forjadora de una cultura de paz que afirme la identidad nacional sustentada en la diversidad cultural, étnica y lingüística, supere la pobreza e impulse el desarrollo sostenible del país y fomente la integración latinoamericana teniendo en cuenta los retos de un mundo globalizado.

De esta forma, podemos advertir que la educación es una actividad indispensable para el progreso y subsistencia en sí de la sociedad misma, y como tal su condición de derecho humano, de derecho fundamental, resulta incuestionable.

Sin embargo, la realidad de la sociedad nos ofrece diversas circunstancias en las cuales este importante derecho no llega a concretizarse, quedando postergado y afectando de este modo a los principales destinatarios de este derecho, los niños en formación en todas partes del mundo. Dentro de las situaciones que limitan el pleno ejercicio de este derecho encontramos a los conflictos armados, los cuales siguen teniendo amplia presencia en el siglo XXI tal como exponemos a continuación.

\section{Los conflictos armados en el siglo XXI}

Hablar de los conflictos armados nos remite a una categoría amplia e inagotable que se asocia con el término genérico de guerra. Sin embargo, en las últimas décadas, y con 
la progresiva consolidación del Derecho Internacional Público y el Derecho Internacional Humanitario, han ido definiéndose criterios más específicos para el tratamiento de este fenómeno, el cual es evaluado desde diversas disciplinas.

Con relación a un concepto o definición, éste tendrá, a nuestra consideración, matices derivados de la connotación que se pretenda brindar; así, en aspectos económicos, políticos, sociales y religiosos, el concepto de conflicto armado se hará sobre la base de un particular ámbito a destacar y que se vincule funcionalmente con la perspectiva que se piensa desarrollar. Para efectos de la presente investigación optamos por una definición desde el ámbito jurídico, en atención a los términos siguientes:

Dentro del DIH hay una distinción entre dos tipos de conflictos: los conflictos armados internacionales (CAl) y los conflictos armados sin carácter internacional (CANI). En el primero se enfrentan dos o más Estados, y es el tipo de conflicto con más normas. Los conflictos armados sin carácter internacional tienen una regulación más limitada, por suceder dentro de las fronteras de un país (...) (Benavides, 2015, pp. 20-21).

Conforme a lo antes señalado, los conflictos armados corresponden a enfrentamientos, siendo categorizados a partir de los agentes que participan. Podemos tener a los Estados (quienes han sido los protagonistas de forma constante a lo largo de la historia de la humanidad), quienes materializan un conflicto internacional; mientras que su contraparte ubica a posiciones de batalla dentro de un mismo país. En ambos casos se encuentran fricciones, ataques y pretensiones. Conviene recordar en este punto la conocida frase de Clausewitz, "la guerra es la continuación de la política por otros medios" (1984, p. 58).

La humanidad ha presenciado las variaciones y nuevos fenómenos que van surgiendo progresivamente en la escalada de conflictos armados, todo ello sumamente vinculado con el avance tecnológico del armamentismo empleado. De esta forma, se tienen enfrentamiento en diversos tiempos y distintas latitudes, y aunque con el surgimiento de instituciones y mecanismos de tutela ha pretendido menguarse los efectos nocivos de los enfrentamientos, sobre todo contra la población civil, se tiene en la práctica una parcela que se sigue afectando sin poder revertirse, y esto es el derecho a la educación de los niños que se encuentran en el contexto del conflicto.

A partir de ello es que la clasificación de los conflictos armados resulta una tarea particularmente compleja en estos tiempos, donde surgen nuevas propuestas sobre sus consideraciones jurídicas. Así, recomendamos entre otros el análisis desarrollado por Vité (2009), el cual suscribimos en sus conclusiones.

Del mismo modo, respecto de la naturaleza de los conflictos armados, esbozar una propuesta resulta particularmente desafiante bajo el contexto en el cual nos desenvolvemos.
Si bien nuestra presente investigación se centra en los efectos de los conflictos contra el derecho a la educación de niños y niñas en todo el mundo. En este sentido, se advierte que "la naturaleza de los conflictos armados y su repercusión en los niños y las niñas están evolucionando. Los conflictos internos se incrementan al mismo ritmo que la cantidad de grupos armados involucrados en ellos" (Save The Children, 2019, p. 12).

Previo al análisis de este complicado panorama, es pertinente tener en cuenta que en el mundo se siguen presenciando los conflictos armados, tal como se expone en el Informe sobre conflictos, derechos humanos y construcción de paz, en el cual se precisa que:

Durante 2020 se registraron 34 conflictos armados, la misma cifra que el año anterior. La mayoría de los conflictos armados se concentró en África (15) y Asia (nueve), seguidos por Oriente Medio (seis), Europa (tres) y América (uno).

En 2020 se observó un incremento significativo de los conflictos armados de alta intensidad, que representaron casi la mitad de los casos, un $47 \%$ del total (2021, p. 21).

A las conocidas secuelas derivadas de los conflictos armados, tales como los daños materiales, los desplazados y el riesgo permanente contra la vida y seguridad, de los cuales han dado cuenta los desgarradores testimonios de las víctimas, hay que considerar con mayor atención las lesiones contra los derechos de carácter económico social y cultural, toda vez que en estos últimos, donde encontramos el derecho al trabajo, a la salud y a la educación, por mencionar algunos, el reparar las secuelas de los daños ocasionados puede tomar años, décadas y toda una vida, lo que incide en las capacidades sociales de los afectados, limitando las urgentes oportunidades que requieren para poder recuperar lo perdido durante el conflicto.

\section{Avances y logros en el ámbito educativo}

Los procesos educativos se han ido perfeccionando paulatinamente. Gracias a la profesionalización de la labor de los docentes, una mayor conciencia por parte 
de los padres acerca de la importancia de que sus hijos sean matriculados en el sistema educativo y toda una especialización multidisciplinaria orientada a mejorar la labor educativa (arquitectura especializada en colegios, psicología educativa, entidades reguladoras y de fiscalización, informática aplicada a procesos pedagógicos, entre otros) han permitido forjar un servicio educativo que apunta a una formación integral, procurando resultados evidenciables mediante diversos instrumentos e indicadores.

En este sentido, debe resaltarse también que se tiene una válida concepción de la educación como un servicio público, lo cual en absoluto se opone o niega su condición de derecho humano merecedor de la más esmerada tutela y protección. De esta forma, la calidad de servicio público que se viene dando a la educación permite comprometer una mayor responsabilidad de las autoridades a que el servicio no se vea afectado o descontinuado por externalidades u otros factores.

Asimismo, de presentarse situaciones extraordinarias o imprevistas, el desarrollo de estrategias y políticas públicas permiten garantizar una gestión educativa capaz de desenvolverse aun en los contextos más difíciles, asegurando que el servicio educativo se pueda seguir prestando. Un claro ejemplo lo representa la situación surgida por la propagación de la COVID-19; si bien la educación remota podría ser complicada de asimilar por los infantes, el hecho de poder seguir brindando lecciones otorgaba una oportunidad frente a la necesidad de acatar una forzosa cuarentena.

Precisamente, el gran impulso que ha presenciado la humanidad en la ciencia y tecnología en los últimos tiempos está directamente vinculado a la mejora de la educación. Sobre la base de los progresos que los han precedido, las nuevas generaciones, con acceso a un sistema educativo debidamente planificado, han podido encontrar diversas vocaciones para concretar y éstas han repercutido, felizmente, en beneficios para toda la humanidad.

No obstante, la educación, y los logros que se plantean para ésta, pueden verse opacados si no recibe un compromiso decidido por parte de las autoridades, tanto en la gestión de sus gobiernos y que estos no sucumban a la ineptitud, o en el escenario más extremo, sumidos en un interminable perecimiento por guerras, plagas, entre otros, tal como advierte de Grasse Tyson (2016):

La educación es clave: tener líderes bien formados, ilustrados, no corruptibles. En muchas naciones en desarrollo es su propia corrupción la que impide que todo el país crezca como debería. Podría verlo desde una postura muy egoísta y decir que quizás el próximo Einstein se está muriendo de hambre en Etiopía y nunca lo sabrás porque es un niño sin comida.

De lo antes señalado, debe entenderse cuán importante resulta erradicar todo conflicto armado del mundo, y también preservar en la gestión educativa un ambiente sin corrupción u otros factores que pudieran poner en riesgo su propósito. El destino de la humanidad está sumamente ligado a la educación que se brinda, y no se puede permanecer impávida la penosa realidad del daño que provocan los conflictos armados a este derecho.

La educación no puede sucumbir antes situaciones tales como las guerras y delitos, porque el daño no va a ser solo a quienes se vieron truncados de estudiar, sino a la humanidad misma al ver limitados a futuros genios que entre el desarrollo y la supervivencia, se vieron obligados a optar por eso último nada más; ni que decir además que una situación de esta naturaleza contraviene el principio de dignidad humana en todos sus extremos, porque se afecta a los seres más indefensos y como tal, más vulnerables de la sociedad: niños y niñas.

\section{Impacto de los conflictos armados contra el derecho a la educación}

\subsection{Consideraciones generales}

Nuestro trabajo procura poner especial atención al derecho a la educación, y los perjuicios que se generan contra éste a partir del desarrollo de los conflictos armados. Para este análisis es importante tener en cuenta a la comunidad educativa, la misma que se encuentra conformada por los padres de familia, los docentes y los estudiantes, siendo estos últimos la prioridad del sistema educativo y eje central de sus procesos. En este sentido, debe asumirse que:

Los conflictos armados repercuten directamente en la educación de los niños y jóvenes, alterando, debido a la realidad que viven, su escala de valores perdiendo la posibilidad de vivir su niñez o adolescencia encontrándose con un mundo que los golpea, los hace encontrarse con lo más duro de la política interna o internacional, haciéndose cargo de situaciones que son las propias de su edad (Porcelli, 2004).

Ahora bien, notamos que un conflicto armado resulta pluriofensivo en el ámbito del proceso educativo, así lo explica Ospina Garnica, refiriendo lo siguiente: 
En el caso concreto de la educación, los conflictos armados debilitan los sistemas educativos, destruyen infraestructuras e instituciones educativas, producen perdidas, generan lesiones y traumas psicológicos entre estudiantes y profesores, como también traen consigo desarraigo, destrucción de la vida familiar y comunitaria, aumento de la violencia social y de la pobreza, entre otros impactos negativos que paralizan u obstaculizan el proceso de aprendizaje y, por ende, el desarrollo de un país (2015, p. 223).

Los diversos perjuicios que se ocasionan contra el proceso educativo a partir de los conflictos armados pueden verse desde dos ámbitos: por un lado, podemos considerar a los integrantes de la comunidad que la conforma, y las lesiones subjetivas que se configuran; pero también podemos advertir perjuicios materiales e institucionales, desde una mirada objetiva. Sobre ello, podemos resumir los puntos en mención a partir del siguiente cuadro:

\section{Cuadro 1}

\section{Efectos de los conflictos armados en la comunidad educativa}

\begin{tabular}{|c|c|c|}
\hline Padres de familia & Docentes & Estudiantes \\
\hline $\begin{array}{l}\text { No ven satisfecho el derecho de que } \\
\text { sus hijos reciban educación. } \\
\text { No pueden planificar debidamente } \\
\text { su vida familiar y profesional. } \\
\text { Se ven limita d os en Ias } \\
\text { oportunidades que pueden brindar } \\
\text { a sus hijos. } \\
\text { Tienen que asumir, forzadamente, la } \\
\text { enseñanza de los aspectos básicos } \\
\text { de conocimientos y habilidades } \\
\text { sociales, a sus hijos. } \\
\text { Afrontan un severo desconsuelo e } \\
\text { impotencia ante la incertidumbre del } \\
\text { futuro de sus hijos. }\end{array}$ & $\begin{array}{l}\text { El derecho a ejercer su trabajo } \\
\text { como docentes se ve seriamente } \\
\text { mermado. } \\
\text { Sus vidas e integridad se encuentran } \\
\text { en permanente riesgo. } \\
\text { Al verse impedidos de trabajar, no } \\
\text { pueden garantizarse medios de } \\
\text { subsistencia. } \\
\text { Sus proyecciones profesionales y } \\
\text { académicas se ven truncadas. } \\
\text { En los casos más extremos, } \\
\text { son reclutados para actuar en } \\
\text { los conflictos, reduciendo así la } \\
\text { cantidad de docentes disponibles. }\end{array}$ & $\begin{array}{l}\text { Las oportunidades de acceder a la } \\
\text { educación son escasas y en muchos casos } \\
\text { completamente nulas. } \\
\text { La educación que reciben, quienes pueden } \\
\text { acceder a alguna, es de calidad deficiente, } \\
\text { toda vez que el entorno no es seguro para } \\
\text { el aprendizaje. } \\
\text { Quienes ser ven forzados a desplazarse y } \\
\text { migrar terminan alterando su formación y } \\
\text { proyección escolar. } \\
\text { Su formación cultural resulta incipiente, } \\
\text { perdiendo, alejándose y olvidándose de sus } \\
\text { propias raíces. } \\
\text { Al verse expuesto a un reclutamiento } \\
\text { forzado para integrar los grupos armados, } \\
\text { pueden alterar todo su proyecto de vida. }\end{array}$ \\
\hline
\end{tabular}

Fuente: Elaboración propia.

Por su parte, en el ámbito institucional, los conflictos armados repercuten negativamente en el proceso educativo de las siguientes formas:

- La educación pierde presupuestos e inversiones por las partidas que se designan al financiamiento de los conflictos.

- La infraestructura educativa se pierde, sea por daños materiales que llevan a su destrucción o porque comienzan a emplearse para otros fines.

- No hay posibilidad de concretar acuerdos de cooperación con especialistas e instituciones externas para poder enriquecer los procesos de aprendizajes, debido a los riesgos que se afrontan.

- Se deja de considerar a la educación como una política estatal, o al diezmarse sus recursos, la autoridad a cargo del sector reduce su presencia, dejando la actividad aislada.

- La práctica pedagógica, al dejar de efectuarse con regularidad, y sin poder realizar las capacitaciones correspondientes, hace los procesos de aprendizaje débiles e improvisados, restando calidad a la educación.

- Los contenidos que se imparten en el currículo escolar pueden verse alterados, sea por una necesidad de las fuerzas beligerantes que terminan imponiéndose y rescriben la historia y sus necesidades según sus intereses, o también por lo limitado de las opciones y solo pueden abordarse parcialmente los distintos contenidos temáticos.

Lo antes expuesto se centra, principalmente, en las consecuencias de la población civil, pasiva en el marco del conflicto armado que afronta severas secuelas y perjuicios a partir de los 
enfrentamientos que, como hemos visto, pueden tomar muchos años o prácticamente no tener fin (como el caso de Israel y Palestina). Un punto más delicado aún, y que debe tenerse en cuenta, lo representa el caso de los menores que forman parte activa del conflicto como niños soldados, sobre lo cual debe considerarse lo siguiente:

Una de las principales causas de la aparición de un conflicto armado y de la participación de los niños en él es la situación socioeconómica en la que se encuentra un determinado país. Los conflictos descansan a menudo en situaciones de desigualdad en la esfera internacional y de desigualdad dentro de las sociedades, lo que suele llevar aparejadas situaciones de conflicto latente o situaciones que pueden acabar degenerando en conflictos abiertos. Una vez que el conflicto ha estallado, las razones económicas y sociales tienen un peso de relativa importancia en la toma de la decisión por parte de un niño de participar en esa contienda (Gómez, 2000, p. 15).

Asimismo, se advierte que en el marco de los conflictos armados y el perjuicio contra el derecho a la educación que venimos exponiendo, en el caso específico de las niñas hay un perjuicio mayor conforme da fe la prensa que aborda el tema, la cual ha reportado que "Los niños y niñas que viven en zonas afectadas por la guerra y la inseguridad no pueden ir a colegio con normalidad. En el caso de las niñas, esa probabilidad se multiplica por dos" (Barchilón, 2020).

Por su parte, y desde una perspectiva muy importante sobre las acciones que se deben adoptar en el seno de la comunidad internacional sobre el caso, Izquierdo Alberca advierte que:

Aunque la repercusión de los conflictos armados en la educación se ha subestimado, la comunidad internacional tiende a reconocer cada vez más la importancia de incrementar y proteger los derechos educativos incluso en esas circunstancias. De la misma forma que es esencial la tarea educativa en la reconstrucción de un país en al post conflicto, los individuos escolarizados hasta la etapa secundaria contribuyen 3 veces más a la estabilidad y la democracia (2015, p. 12).

Una mención particular radica en los efectos que ha tenido la pandemia originada por la COVID-19 en todo el mundo y como la misma ha terminado afectando aún más la difícil situación que afrontan los estudiantes involucrados en los conflictos armados, dándose cuenta al respecto en el informe anual del secretario general de la ONU sobre menores y conflictos armados publicado en junio de 2020, precisándose al respecto que:

Más allá de las conclusiones del informe del secretario general de la ONU, el análisis de los conflictos armados activos en 2020 confirma el patrón de vulneraciones contra menores, agravados por el contexto de la COVID-19. La pandemia limitó aún más el acceso a determinados derechos como el de la educación. Así, por ejemplo, en el caso de Jammu y Cachemira, en Pakistán, el cierre de las escuelas con motivo de la pandemia se sumó a meses de clausura previa de los centros educativos a causa del conflicto (p. 31).
Tal como venimos detallando, los conflictos armados tienen diversas y severas repercusiones contra los derechos de los niños y niñas, enfocando nuestro análisis en el derecho fundamental a la educación y frente a ello urge la adopción de medidas específicas. A partir de ello se hace mención de una Educación en Emergencia la cual, conforme a Villegas (2011), debe tener en cuenta lo siguiente:

Para fortalecer la implementación de la Educación en Emergencia por el conflicto armado, es ineludible que sea considerada como una necesidad social y política, por lo cual debe estar integrada a la ayuda humanitaria y a la asistencia para el desarrollo, ya que el refugio, la nutrición, la salud y la educación son derechos interdependientes (p. 50).

Como una fuente especial para el estudio de los problemas que debe hacer frente la educación en general desde diversas latitudes, podemos recurrir a los aportes realizados por la UNESCO mediante los estudios que impulsa y en los cuales se abordan los problemas que afronta la educación. Mediante informes periódicos se da cuenta acerca de los resultados de las diversas problemáticas consideradas. A manera de muestra sobre ello, podemos invocar los siguientes:

- 2002: Educación para todos - ¿Va el mundo por el buen camino?

- 2003/4: Educación para todos - Hacia la igualdad entre los sexos

- 2005: Educación para todos - El imperativo de la calidad

- 2006: Educación para todos - La alfabetización, un factor vital

- 2007: Bases sólidas - Atención y educación de la primera infancia

- 2008: Educación para todos en 2015 ¿Alcanzaremos la meta?

- 2009: Superar la desigualdad: por qué es importante la gobernanza

- 2010: Llegar a los marginados

- 2011: Una crisis encubierta: Conflictos armados y educación

- 2012: Los jóvenes y las competencias: Trabajar con la educación

- 2013/4: Enseñanza y aprendizaje: Lograr la calidad para todos 
- 2015: La Educación para Todos 2000-2015: Logros y desafíos

- 2016: La educación al servicio de los pueblos y el planeta: Creación de futuros sostenibles para todos

- 2017/8: Rendir cuentas en el ámbito de la educación: Cumplir nuestros compromisos

- 2019: Migración, desplazamientos y educación: Construyendo puentes, no muros

- 2020: Inclusión y educación: Todos y todas sin excepción.

En este sentido, consideramos como una fuente indispensable para el estudio de los problemas que inciden en el derecho a la educación estos valiosos informes, los mismos que ratifican las diversas amenazas que se erigen en contra de estos derechos a partir de distintos factores. Para el caso de la presente investigación, merece especial atención el informe de 2011, en el cual se resumió en gran medida las consecuencias de los conflictos armados en el ámbito educativo, destacándose lo siguiente:

- Deficiencias en la protección. Los gobiernos nacionales no actúan basándose en sus responsabilidades éticas, ni tampoco en sus obligaciones jurídicas, para proteger a los civiles que se ven atrapados por conflictos armados. Existe toda una cultura de la impunidad en torno a atentados atroces perpetrados contra los derechos humanos, que constituye un obstáculo considerable para la educación (...).

- Deficiencias en la prestación de servicios educativos. Los padres y los niños afectados por conflictos armados muestran una firmeza extraordinaria en su determinación por mantener a toda costa la educación en circunstancias adversas (...).

- Deficiencias en la recuperación y reconstrucción iniciales. Los convenios de paz ofrecen a los gobiernos de países en situaciones posteriores a conflictos, así como a la comunidad internacional, la posibilidad de elaborar y aplicar estrategias de recuperación y reconstrucción (...).

- Deficiencias en la construcción de la paz. La educación puede desempeñar un papel esencial en la construcción de la paz. Es posible que sea en este sector donde la educación puede conseguir los beneficios iniciales de la paz más visibles, de los que depende la supervivencia de los acuerdos de paz (...) (p. 26).

\subsection{El caso peruano}

En el Perú las situaciones de conflicto armado internacional han sido específicas, siendo el recuerdo más funesto el de la ocupación de la capital y otras ciudades en el contexto de la Guerra del Pacífico (1879-1883). Como se sabe, las secuelas del conflicto fueron diversas, con innumerables bajas, saqueos y un considerable daño al patrimonio histórico del país. Si bien entonces no había un concepto pleno de la educación como derecho fundamental, es sumamente conocido el arrojo de estudiantes del Colegio Nuestra Señora de Guadalupe y otras escuelas durante la defensa de Lima, perdiéndose la vida heroica y trágicamente.
Sin embargo, en el caso de los conflictos armados al interior del país, los hechos desarrollados por la agrupación terrorista Sendero Luminoso provocaron un daño al derecho fundamental a la educación de proporciones mayúsculas, tanto a los miembros de la comunidad educativa como a la infraestructura y posibilidades para el sector. Los efectos de este insano accionar aún se siguen sufriendo a pesar de las décadas transcurridas, y con la constante amenaza del rebrote de ese grupo o sus aliados en lugares focalizados del Perú, donde su presencia no ha podido ser desterrada.

Precisamente, en el presente siglo XXI, viene siendo un problema vigente la presencia de conflictos armados internos en el país, los cuales tienen como epicentro la zona del VRAEM (Valle de los ríos Apurímac, Ene y Mantaro) donde tienen a lugar operaciones terroristas y de narcotráfico que han originado declarar el lugar en permanente estado de emergencia, precisándose al respecto:

Según Alonso Gurmendi, en el caso concreto, este reconocimiento por parte del Estado se dio por primera vez a través del Decreto Supremo 076-2016-PCM, indicando explícitamente, en su exposición de motivos, que existe un conflicto armado con el Militarizado Partido Comunista del Perú (MPCP). Dicho Decreto Supremo fue prorrogado por el Decreto Supremo 075-2021-PCM (Meza, 2021).

Hablar del VRAEM en remitirnos a una zona compleja, foco de diversos problemas y como tal muy delicada en su tratamiento y las medidas a aplicarse. Por sus condiciones inseguras gran parte de su realidad solo puede conocerse vía los medios de prensa, los cuales han informado que:

Es una de las zonas más deprimidas del país, con altos índices de pobreza extrema, precarios servicios de comunicación y graves déficits de salud y educación. Su economía está poco diversificada y es fundamentalmente agrícola, destacando cultivos como el cacao, el café y la hoja de coca, materia prima de la producción de cocaína (Cueto, 2021).

Asimismo, conforme a distintos reportes, existe dentro de la zona del VRAEM una fuerte presencia de los remanentes del grupo terrorista Sendero Luminoso (quienes 
provienen del Militarizado Partido Comunista del Perú), quienes actúan en esta región con el propósito de controlar los cultivos de hoja de coca, impulsando el narcotráfico y creando alianzas con quienes se encuentran vinculados con esta nefasta actividad. En este sentido, se advierte que el modus operandi de dicho grupo en la región es contar con un espacio para el cultivo y fomento de la hoja de coca, insumo esencial para la producción de cocaína, y por ende, con amplia incidencia en el tráfico de drogas.

La problemática que se yergue en el VRAEM, sobre el derecho fundamental a la educación, ya ha sido materia de atención en ciertos trabajos, incidiéndose en la pluralidad de lesiones que se configuran contra los miembros de la comunidad educativa y que realmente constituye una dramática situación que no se difunde con la urgencia que demanda, conforme se advierte de lo siguiente:

El Valle del Río Apurímac, Ene y Mantaro (VRAEM) es una de las zonas más convulsionadas del Perú, donde el Estado lucha incansablemente contra el narcotráfico y el terrorismo. Sin embargo, hay otra lucha diaria que libran centenares de educadores de esa zona, tratando de evitar que los jóvenes caigan en la trampa de la violencia y piden mejorar las condiciones educativas en sus colegios (Programa Nacional de Infraestructura Educativa, 2018)

Como tal, y teniendo en cuenta lo expuesto, los conflictos armados son una realidad, y especialmente en el caso del Perú, tenemos aun situaciones por resolver que rebasan la propia configuración de los enfrentamientos y las bajas que terminan suscitándose de ambos bandos; tan grave como la situación beligerante viene a ser el daño contra los derechos sociales de la población civil que se ve forzadamente aprisionada del conflicto.

La multiculturalidad de nuestro país, si bien lo hace especial y complejo, también ha generado indolencia en ciertos espacios en los cuales los problemas de estos sectores de la provincia y los valles no ocupan mayor atención del gran público de las ciudades. Si bien periódicamente en el Perú se presentan emergencias derivadas de situaciones climatológicas como sequías, huaycos y heladas a lo largo y ancho del país, la permanente situación del VRAEM no concita la atención que merece, salvo en contados episodios, pero que luego pierden cobertura mediática como lo ocurrido el 24 de mayo de 2021 en el contexto de las elecciones presidenciales peruanas (Canal N, 2021).

Consideramos que persiste un descuido en entender, por parte de las autoridades, los factores esenciales y estructurales que nutren el conflicto en la zona del VRAEM. La sola estrategia militar para el lugar no va a garantizar mejoras para la población civil que termina viviendo a merced de otros, sin propia voluntad ni seguridad. Es indispensable rescatar a la población, pero no en términos físicos, sino en reforzar la experiencia educativa y en paralelo promover el diálogo, para que puedan desprenderse de las condiciones que los apresan tanto hoy como para el futuro.

$Y$ en este sentido urge difundir más estudios y reportajes en torno a la complicada situación que afrontan los peruanos que habitan en dicha región, para poder brindarles soluciones y oportunidades que requieren y merecen; aun con los esfuerzos militares por enfrentar la presencia del narcotráfico y el terrorismo en el lugar, no se está tomando en cuenta la real magnitud del conflicto armado respecto de los perjuicios que se configuran contra otros derechos fundamentales. Hemos destacado en el presente estudio el caso del derecho a la educación, pero también pueden advertirse otros tales como el derecho al trabajo, el derecho a gozar de un ambiente equilibrado y adecuado al desarrollo de la vida, el derecho de propiedad, entre otros.

Asimismo, debe tenerse en cuenta que remediar las lesiones contra los derechos de carácter social, producidas debido a los conflictos, pueden resultar más complicadas de atender por lo progresivo del desarrollo de las personas afectado. Generalmente la niñez es la etapa por excelencia para la educación básica de las personas, y frente ello, recuperar el tiempo perdido es casi inviable, con lo cual además de poder brindar acceso a la educación formal u alternativa, también consideramos indispensable dotar de un acompañamiento psicológico apropiado que asegure a los involucrados una orientación que les permita integrarse con éxito a la sociedad en su conjunto.

En general, la atención de los derechos fundamentales de las víctimas de los conflictos armados, exige también un replanteamiento del derecho a la igualdad de las personas, una de las piedras angulares del Estado Moderno, teniéndose en cuenta que la igualdad no se trata de brindar tratos idénticos sin miramientos, sino el derecho de tratar de igual modo a quienes se encuentran en una idéntica situación, criterio concordante con lo expuesto por el Tribunal Constitucional peruano (Sentencia recaída en el Expediente 00009-2007-PI/TC). 
Por lo tanto, aun con la complejidad y factores propios de cada conflicto armado que se produce el día de hoy, o en el futuro, es indispensable adoptar medidas efectivas frente a los agravios contra el derecho fundamental a la educación, toda vez que conforme se ha expuesto, las secuelas dejadas repercuten y tienen especial impacto durante toda la vida de los menores involucrados en el conflicto, y hasta puedan cerrar toda perspectiva y esperanza sobre lo que la educación realmente representa para el futuro de la humanidad misma.

Dentro del desarrollo de la presente investigación, hemos podido reparar que existen una serie de estudios, multidisciplinarios, los cuales, a partir de los problemas para el derecho a la educación en el marco de los conflictos armados, abordan diversos efectos que surgen por estos casos, lo cual en definitiva constituye un interesante eje de reflexión que debe seguir progresando en su inquietud y análisis.

Incluso, los enfoques que hemos mencionado pueden partir de acontecimientos bélicos del pasado, pero rescatando los alcances que pueden generar interés en otras ciencias tales como la psicología y la pedagogía, por mencionar algunos, tal como se desprende del trabajo de Vicent et al. (2020) en el cual refieren que:

Durante la fase de cribado de la muestra, se ha constatado que una de las consecuencias de los conflictos armados -caso, por ejemplo, de las guerras mundiales-, es que suelen suponer un hito temporal a partir del cual la educación para la ciudadanía y la enseñanza de las ciencias sociales son reformuladas. Ambos campos educativos son delicados y exigen prestar atención al modo en el que se abordan en las aulas (p. 75).

Es evidente que conocer el nivel de agravio de los conflictos armados contra la comunidad educativa y el derecho fundamental a la educación demanda la oportunidad de acercarse a los involucrados y testigos en estas situaciones para poder tener un real diagnóstico de las secuelas que se manifiestan y a partir de ella establecer políticas mucho más efectivas para brindar respaldo a quienes tuvieron que vivir el flagelo de la violencia y ver postergado su proyecto de vida.

A partir de ello, consideramos que la comunidad internacional y las instituciones deben organizar en gran medida el trabajo que pueda realizarse para atender a quienes se vieron afectados por los conflictos armados. Asumimos que la mejor forma de desarrollar un trabajo con expectativas fructíferas es articulando esfuerzos y teniendo un respaldo estructurado para que se pueda llegar a la mayor cantidad de personas que requieran ayuda, y como tal, los recursos que sustenten dicha labor puedan ser constantes teniendo en cuenta que la finalidad es rescatar a la población inocente de la violencia desmedida que surge y que no repara en el desastre que ocasiona.

\section{Conclusiones}

La educación a lo largo de la historia ha favorecido el progreso humano, y los efectos en la profesionalización de sus agentes y la modernización de sus procesos permitieron un avance sin precedentes en el último siglo en el ámbito de las ciencias en todo nivel.

El derecho humano a la educación evidencia ese manifiesto reconocimiento, por parte de los pueblos del mundo, a la importancia de la actividad educativa, siendo indispensable garantizar su acceso, prestación de calidad y procurando el respeto al estudiante en formación.

Los conflictos armados han sido una constante en la historia de la humanidad, afrontando diversas etapas y momentos. Lamentablemente la paz aún está lejos de ser una realidad para todos los rincones del globo, y debe tomarse especial atención en los derechos que resultan especialmente afectados producto de estos enfrentamientos.

Un conflicto armado origina serias y diversas lesiones a los miembros de la comunidad educativa, propiamente constituye una situación pluriofensiva donde los estudiantes son sumamente perjudicados, pero en el caso de los padres de familia y docentes también afrontan negativas repercusiones que pueden tener impacto a lo largo de su vida.

No debemos dejar de notar la situación particular en el Perú, donde aún en ciertas regiones hay conflictos derivados de la lucha contra los remanentes del terrorismo y del narcotráfico. Sumando a la precaria situación de la infraestructura educativa en varias localidades, en regiones como el VRAEM el drama que afrontan los estudiantes los condena a un círculo vicioso en el cual, al no tenerse a la educación como opción, se verán forzados a someterse al accionar ilícito de mantenerse las condiciones como hasta ahora.

Si bien a nivel del gobierno peruano se han adoptado algunas acciones para recuperar al ámbito educativo en las zonas de conflicto, consideramos indispensable que la realidad que se afronta sea difundida 
a nivel nacional con el objeto de que tome real conciencia de los perjuicios que se generan producto de la violencia, y que la ciudadanía y las autoridades puedan prevenir este tipo de conductas en sus localidades.

La educación seguirá siendo el patrimonio humano por excelencia, así como la fuente para preservar su desarrollo y supervivencia; que los conflictos armados no sigan agraviando a inocentes que se ven imposibilitados de continuar sus estudios debe ser una preocupación constante y prioritaria en todo plano de decisión nacional e internacional.

\section{Referencias bibliográficas}

Barchilón, M. (2018, 13 de setiembre). Cuando la guerra pone en peligro la educación. La Vanguardia. https://www.lavanguardia.com/ vida/junior-report/20180913/451776332296/educacion-peligro-guerraconflicto-armado.html

Benavides, L. (2015). Derecho Internacional Humanitario. Comisión Nacional de los Derechos Humanos.

Canal N. (2021, 24 de mayo). Vraem: Terroristas asesinan a 14 personas en el distrito de Vizcatán del Ene. https://canaln.pe/ actualidad/vraem-terroristas-ejecutan-al-menos-18-personasvizcatan-ene-n435350?ref=

Chanamé, R. (2011). La Constitución Comentada. Editorial ADRUS.

Clausewitz, K. (1984). De la guerra. Labor.

Cuenca, R. (2012). Desencuentros entre el discurso del derecho a la educación y las políticas educativas en el Perú de la década del 2000. IEP-Clacso.

Cueto, J. (2021, 25 de mayo). Perú: cómo es el Vraem, el "valle de la droga" en el que ocurrió la masacre de 16 personas atribuida a Sendero Luminoso. BBC News. https://www.bbc.com/mundo/noticias-americalatina-57240760

Degrasse, N. (2016, 30 de junio). Quizás el próximo Einstein se está muriendo de hambre en Etiopía. El País. https://elpais.com/ elpais/2016/06/30/ciencia/1467281442_280683.html

García, V. (2008). Los derechos fundamentales en el Perú. Jurista Editores.

Gómez, F. (2000). La participación de los niños en los conflictos armados. El Protocolo Facultativo a la Convención sobre los Derechos del Niño. Universidad de Deusto.

Izquierdo, J. (2015). Escuelas y Educación en los Conflictos Armados (DIEEEA19-2015). Instituto Español de Estudios Estratégicos (IEEE). https://www.ieee.es/Galerias/fichero/docs_analisis/2015/ DIEEEA19-2015_Escuelas-Educacion_ConflictosArmados_MJIA. pdf

Meza, C. (2021). VRAEM: ¿Estamos ante un conflicto armado o terrorismo? Ventana Jurídica Facultad de Derecho PUCP. https:// facultad.pucp.edu.pe/derecho/ventanajuridica/vraem-estamos-anteun-conflicto-armado-o-terrorismo/
Navarro, I., Royo, J., Urgell, J., Urrutia, P., Villelas, A. \& Villelas, M. (2021). Alerta 2021! Informe sobre conflictos, derechos humanos y construcción de paz. Escola de Cultura de Pau.

Nicoletti, J. (2009). La educación como derecho universal. EDUCATIO Revista Regional de Investigación Educativa, 7, 73-85.

Ospina, J. (2015, 30 de junio). La educación para la paz en situaciones de conflicto armado. Construyendo un nuevo enfoque desde el caso de los Territorios Palestinos Ocupados. [tesis doctoral, Universidad Carlos III de Madrid. Instituto de Derechos Humanos Bartolomé de las Casas]. Biblioteca Universidad Carlos III de Madrid. http:// hdl.handle.net/10016/21935

Ospina, J. (2016). El derecho a la educación en situaciones de conflicto armado: de las manifestaciones e impactos de la violencia a la construcción de la paz. Universitas. Revista de Filosofía, Derecho y Política, 209-242.

Porcelli, E. (2004, 11 - 12 de noviembre). Niñez en conflictos armados: La educación no formal, una alternativa para la paz. II Congreso en Relaciones Internacionales del IRI - La Plata, Argentina. Universidad Nacional de la Plata.

Programa Nacional de Infraestructura Educativa. (2018, 11 de junio). La educación se abre paso en comunidades del VRAEM con nuevos proyectos educativos. https://www.gob.pe/institucion/pronied/ noticias/208860-la-educacion-se-abre-paso-encomunidades-del-vraem-con-nuevos-proyectoseducativos

Save the Children. (2019). No a la guerra contra la infancia. https://www.savethechildren.es/sites/ default/files/imce/docs/informe_no_a_la_guerra_ contra_la_infancia.pdf

Tribunal Constitucional. (2005, 18 de febrero). Sentencia recaída en el expediente 0091-2005PA/TC.

Tribunal Constitucional. (2007, 29 de agosto). Sentencia recaída en el expediente 00009-2007$\mathrm{PI} / \mathrm{TC}$.

Tribunal Constitucional. (2007, 17 de octubre). Sentencia recaída en el expediente 4646-2007$\mathrm{AA} / \mathrm{TC}$.

UNESCO. (2011). Informe de Seguimiento de la Educación para todos en el Mundo. París.

Vicent, N., Castrillo, J., Ibañez-Etxeberria, A., \& Albas, L. (2020). Conflictos armados y su tratamiento en educación. Análisis de la producción científica de los últimos 25 años en la Web of Science. Revista digital de Historia y Didáctica de la Historia, 14(2), 55-91. 
Villegas, M. (2011). Niños, niñas y jóvenes afectados por el conflicto armado, Educación en Derechos Humanos y Derecho a la Educación [tesis de grado de maestría en psicología comunitaria, Pontificia Universidad Javeriana]. Repositorio institucional de la Pontificia Universidad Javeriana. https://repository.javeriana.edu.co/bitstream/handle/10554/1550/ VillegasPati\%C3\%B1oMarcela2011.pdf?sequence $=5$

Vité, S. (2009). Tipología de los conflictos armados en el derecho internacional humanitario: conceptos jurídicos y situaciones reales. International Review of the Red Cross, 873, 69-94. (8010) 